\title{
A Survey on Smart Capture
}

\author{
Vilas Rathod ${ }^{1}$, Soham Kulkarni ${ }^{2}$, Rutuja Paygude ${ }^{3}$, Samruddhi More ${ }^{4}$, Akash Bhosale ${ }^{5}$ \\ Guide Professor, Department IT, MIT Polytechnic, Pune, India ${ }^{1}$ \\ Student, Department IT, MIT Polytechnic, Pune, India ${ }^{2,3,4,5}$
}

\begin{abstract}
If we imagine shooting of movie the first picture comes in the mind with big trolleys loaded with a camera and a camera man with it. Rolling, Rotating, Capturing a perfect angle needs a huge space and a lot of hard work to shoot even a 5 minute seen. The Smart Capture is being introduced to get easier way.
\end{abstract}

Keywords: Smart Shooting, Raspberry Pi, Remote Access, Smart Control

\section{INTRODUCTION}

To easier the shooting process in a compact way with a smart vision and laser physical work the system is being introduce named as "Smart Capture". The system allows user to control camera remotely with the help of website and even through mobile using an application. The camera not only just captures from different angles but also it moves and captures from different locations.

\section{LITERATURE SURVEY}

Among the existing surveillance techniques, CCTV is the most commonly used one. But it has its own limitations. It is a passive monitoring device and it needs continuous human intervention for monitoring. The investigation is a little bit hectic thing since all the previously recorded videos need to be watched manually. Moreover files can be corrupted very easily and this technique is costly too. These limitations lead to the development of active surveillance system. Several researchers have come up with the idea of active surveillance systems in various papers. Most of the paper utilizes the advantage of Wireless Sensor Networks (WSN) for surveillance. Since the sensor nodes being wireless, they can be placed any where inside the building, thus it achieves portability in deployment.

[1]. This paper describes the WSN based surveillance system monitored by Programmable System on Chip (PSOC) devices. Here Zigbee module is used for wireless transmission. The system mainly concentrates on sensor based alerts and it lacks improved techniques like camera, web server for uploading files etc.

[2]. This paper describes an improved real time home security system using BeagleBoard and Zigbee Remote alert on fire and intruder detection are the main features of the system. It uses improved techniques such as camera, GSM, FTP server etc. But it is not utilizing the advantage of live streaming and alerting techniques such as phone calls, SMS and email etc.

[3]. This paper describes the Internet of Things approach for motion detection using Raspberry Pi. It utilizes FTP server for camera feeds and it alerts user through email. The system does not have SMS and phone call alerts and other sensor alerts such as detection of fire, gas etc.

[4]. This paper explains a WSN based fire alarm system using Arduino. The system is purely based on sensor alerts and it lacks other features like camera, web server etc.

[5]. This paper describes the surveillance technique using IP camera and Arduino board. In this paper, user can view remote desktop using team viewer application whenever he needs to monitor his home from outside. This system is not sending any notification to user whenever any event occurs in his home. User has to monitor his home continuously and also it lacks sensor based alerts.

[6]. This paper describes the security surveillance system using Raspberry Pi. It utilizes the advantage of live streaming. But we need to type the IP address every time to watch the live video which is placed in webpage.

\section{Problem Definition}

To introduce a system which can be operated remotely using website and android application to shoot by a camera on location. Not only can capture the things but also controlled by owner remotely. 


\section{EXISTING SYSTEM}

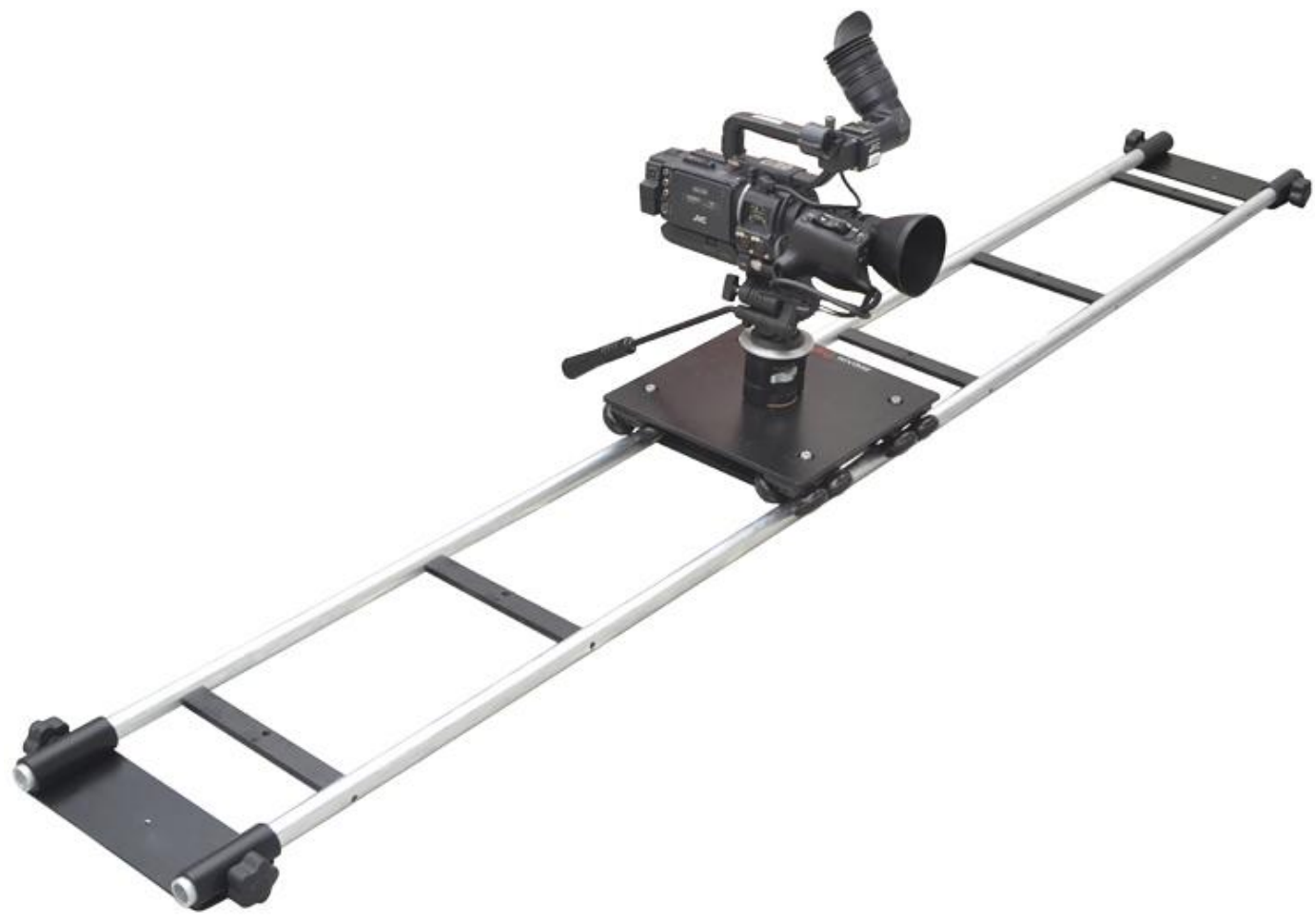

FIG 1 EXISTING SYSTEM

\section{Propose System}

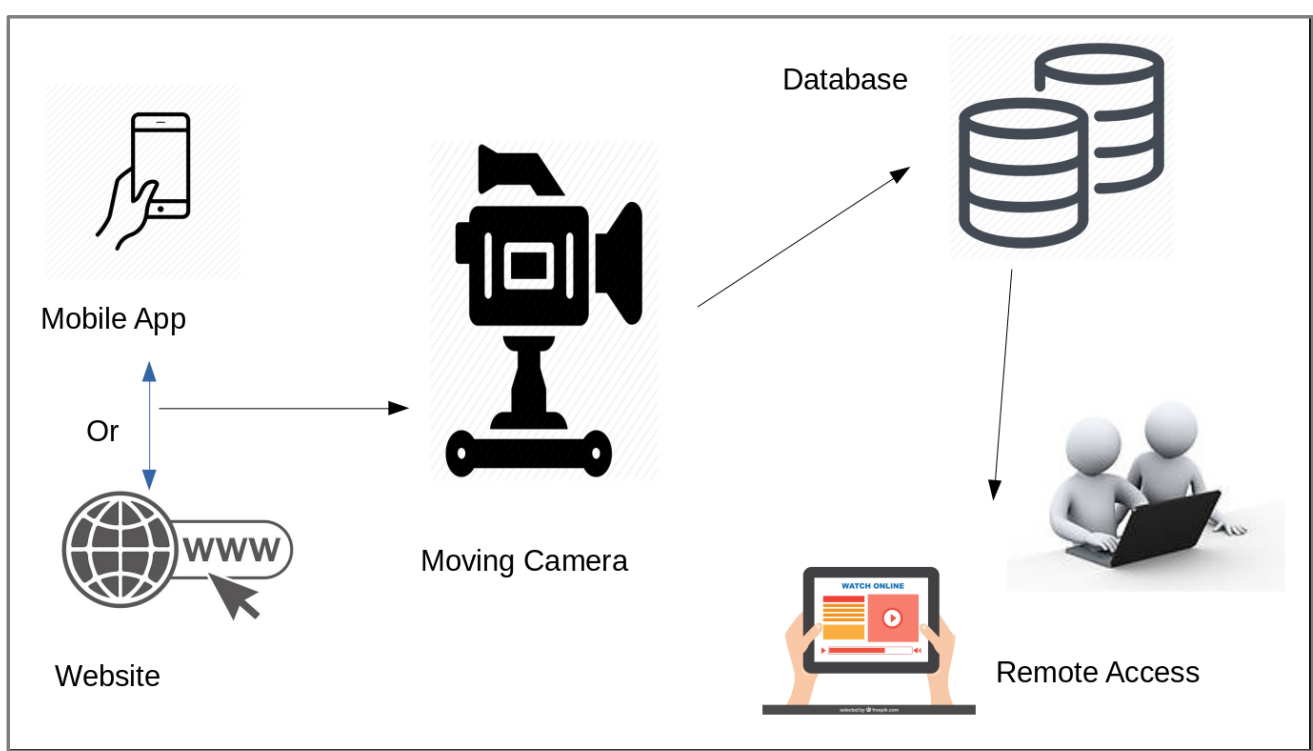

FIG 2 SYSTEM ARCHITECTURE OF SMART CAPTURE 


\section{IJARCCE}

Vol. 8, Issue 2, February 2019

\section{SYSTEM ALGORITHM}

1. Start.

2. User gives input to the system via Website or android application.

3.The input is fetched by Raspberry Pi using network.

4.The commands by user are processed by rasberry pi.

5. Raspberry Pi gives controls the camera module.

6.Camera module moves and shoot or capture according to the user command.

7.The result is stored in database.

8.The result is shown to user via Database.

9.Stop.

\section{FUTURE SCOPE}

1.The more Authentications will be provided.

2.Size will be more compact.

3.New filters will be added to the system.

4clarity of camera is better than Existing.

\section{CONCLUSION}

After the deep study the proposed system Smart Capturer can be introduce by detailing with appropriate hardware and software combination.

\section{REFERENCES}

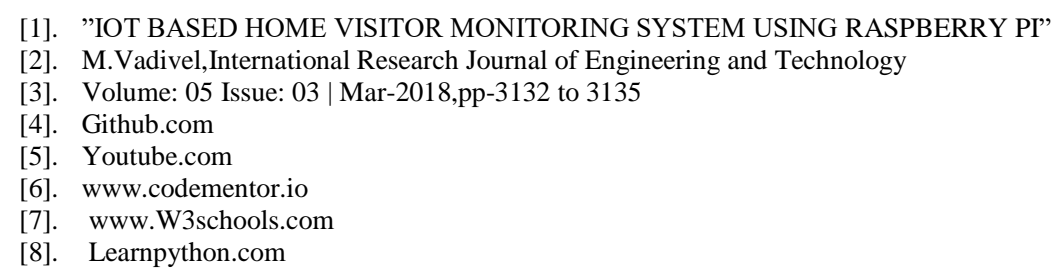

